\section{RNA DEGRADATION}

\section{Imaging nonsense}

Mol. Cell https://doi.org/10.1016/j.

molcel.2019.05.008 (2019)
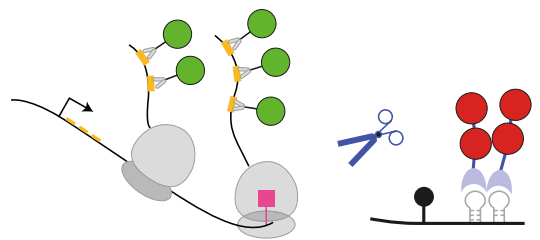

Credit: Elsevier

Transcripts carrying nonsense mutations undergo rapid degradation via nonsensemediated mRNA decay (NMD) to prevent accumulation of toxic truncated peptides. Previous studies have shown that mRNA molecules with different sequences exhibit NMD with varying efficiency, but a lack of useful detection methods makes it challenging to reveal the underlying mechanisms. To precisely determine the cleavage kinetics of NMD, Hoek et al. developed a method to visualize the translation and degradation of single mRNA molecules carrying premature termination codons (PTCs) in live cells. They introduced fluorescent tags at the $5^{\prime}$ and $3^{\prime}$ ends of a reporter transcript and observed the exact moment of NMD cleavage for each molecule, as this resulted in separation of the two fluorescence signals. Using this system, they found that the cleavage rate was affected by exon sequences downstream of the PTC, the distance of the PTC to downstream introns, and the number of introns. In addition, they uncovered the kinetics by which XRN1 degrades the $3^{\prime}$ cleavage fragments produced by NMD. This study provides a useful tool to investigate the kinetics of RNA degradation and deepens our mechanistic understanding of NMD. YS

https://doi.org/10.1038/s41589-019-0333-3

\section{CHEMICAL GENETICS}

\section{PROSPECTing for drugs}

Nature https://doi.org/10.1038/s41586-019-1315-z (2019)

The use of whole-cell and biochemical antibiotic screens to identify novel smallmolecule inhibitors to treat Mycobacterium tuberculosis $(M t b)$ infections has been largely unsuccessful because of the inability to comprehensively target a large number of essential proteins. Johnson et al. developed a drug discovery approach called PROSPECT (primary screening of strains to prioritize expanded chemistry and targets) as an alternative. PROSPECT involves screening compounds against haploid bacterial genetic mutant strains depleted of 474 essential $M t b$ genes mainly through conditional proteolysis. Screening pools of 100-150 diverse strains, each containing a unique barcode, against bioactive and unbiased compound libraries generated 8.5 million chemical-genetic interactions to test whether compounds specifically targeted a particular mutant strain over wild-type strains. This approach identified 40 compounds targeting various processes ranging from DNA gyrase to tryptophan biosynthesis. In particular, they identified BRD-8000 as an uncompetitive direct inhibitor of the efflux pump EfpA. Overall, the PROSPECT

\title{
GENOME EDITING
}

\section{DNA jumps with CRISPR Science https://doi.org/10.1126/science.aax9181 (2019)}

Some CRISPR-Cas variants with deficient nuclease activity are reported to associate with Tn7-like transposons. However, whether these variants are functional in DNA transposition is unclear. Strecker at al. characterized two CRISPR-associated transposase (CAST) loci from the cyanobacteria Scytonema hofmanni and Anabaena cylindrica and found that four proteins (TnsC, TnsB, TniQ, and Cas12k) encoded in both CASTs are essential to mediate DNA transposition in vitro and in Escherichia coli with the assistance of tracrRNA and crRNA targeting a synthetic protospacer. Sequencing analysis revealed that both CASTs have a preference for NGTN protospacer adjacent motifs (PAMs), and DNA insertions localize within a small window downstream of the PAMs. Further studies showed that the S. hofmanni CAST can mediate transposition of DNA segments as long as $10 \mathrm{~kb}$ and insertion of endogenous DNA in the E. coli genome with a frequency of up to $80 \%$. This study enriches the diversity of the CRISPR toolbox and paves the way for developing gene knock-in tools with high efficiency.

https://doi.org/10.1038/s41589-019-0335-1

system offers a promising drug discovery approach that enables identification of compounds that can act on a large range of potential $M t b$ targets.

https://doi.org/10.1038/s41589-019-0334-2

\section{MEMBRANE-PROTEIN INTERACTIONS Freedom to bind}

J. Am. Chem. Soc. https://doi.org/10.1021/ jacs.9b03927 (2019)

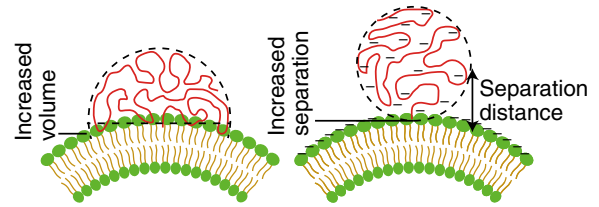

Credit: ACS

Some intrinsically disordered protein domains have recently been found to sense the curvature of membranes, such as those in endocytic pits and tubular organelles. To define the mechanisms by which disordered domains sense membrane curvature, Zeno et al. tested a series of disordered segments within the C-terminal domain (CTD) of the endocytic protein AP180 for binding to small vesicles of increasing diameter (decreasing curvature). They first found that decreasing the chain length of the AP180 segment reduced curvature sensitivity, consistent with the lower conformational entropy of shorter chains. However, the decrease in free energy was weaker than would be expected if curvature sensing arises only from increases in conformational entropy, hinting that electrostatic effects could also be in play. By varying the ionic strength of the solution, the authors could indeed tune the degree of repulsion between the negatively charged lipids of the vesicles and the substantial net negative charge in AP180CTD to conclude that minimizing electrostatic effects can drive curvature sensing. These results suggest an interplay between entropic effects that are driven by reducing restriction on protein conformation and electrostatic effects to minimize protein-membrane repulsion in sensing membrane curvature.

https://doi.org/10.1038/s41589-019-0332-4

Mirella Bucci, Grant Miura and Yiyun Song 